Bull. Mater. Sci., Vol. 22, No. 3, May 1999, pp. 697-700. (C) Indian Academy of Sciences.

\title{
In situ TEM observation of long range ordering via short range order in $\mathrm{Cu}_{3} \mathbf{P t}$
}

\author{
N KUWANO*, N CHIWATA and K OKI \\ Department of Applied Science for Electronics and Materials, Interdisciplinary Graduate School of Engineering \\ Sciences, Kyushu University, Kasuga, Fukuoka 816-8580, Japan
}

\begin{abstract}
The thermoequilibrium state of short range order (SRO) and the ordering process to the long range order (LRO) phase in $\mathrm{Cu}_{3} \mathrm{Pt}$ have been studied by the in situ transmission electron microscope (TEM) observation method. It is confirmed that the alloy has the SRO state in thermoequilibrium within the temperature region for the disordered fcc phase (designated as A1). The SRO state can be interpreted in the 'microdomain model'. The degree of order fluctuates temporally as well as spatially at higher temperature, but freezes at a lower temperature in the $A 1$ region. In the temperature region of $(A 1+L R O)$, some of the microdomains in the SRO grow up to large domains of LRO. This indicates that the transformation process to LRO via SRO proceeds without a process of nucleation even though the transformation is of a first order. Division of A1 into subregions and physical meaning of the phase boundary between (A1 + LRO) and $A 1$ are discussed.
\end{abstract}

Keywords. In situ TEM observation; short range order; ordering kinetics; first order transformation; phase diagram; copper based alloy.

\section{Introduction}

Many Cu-based alloys quenched from a high temperature region for an $\mathrm{fcc}(\mathrm{Al})$ phase show characteristic diffuse scatterings in their diffraction patterns. The origin of the diffuse scatterings is explained by the Fermi-surface imaging (Krivoglaz 1969; Moss 1969; Ohshima and Watanabe 1973). It has been reported that dark-field image taken by using the diffuse scattering frequently indicates a fine dot-contrast (Torfs et al 1974, 1975; Chevalier and Stobbs 1979; Kuwano et al 1987; Lee et al 1990). The diffuse scattering is attributed to the presence of short range order (SRO) and the fine dotcontrast is considered to be an evidence for 'microdomains'. In this connection, Aubauer (1972) proposed that microdomains dispersed in the disordered matrix are stabilized by the elastic energy between the ordered microdomains and the disordered matrix. However, the possibility that the microdomains observed in the dark field image might have been actually formed during quenching cannot be completely excluded if the experiment is carried out for quenched specimens. In this study, we carried out in situ transmission electron microscopic (TEM) observations in order to clarify if the microdomains are stabilized in $\mathrm{Al}$-phase of $\mathrm{Cu}_{3} \mathrm{Pt}$ and to analyze the early stage of ordering process from SRO. The phase diagram including the SRO state and the physical meaning of phase boundary of $\mathrm{A} 1 /(\mathrm{A} 1+\mathrm{LRO})$ are discussed.

*Author for correspondence

\section{Experimental}

An alloy ingot was made from pure metals of copper (99.99\% pure) and platinum $(99.95 \%$ pure) in an arcfurnace under an atmosphere of argon. Disk specimens $3 \mathrm{~mm}$ in diameter for TEM observation and powder specimen for X-ray diffraction experiment were prepared from the ingot after annealing for homogenization. The chemical composition of the alloy was estimated to be 25.1 at $\% \mathrm{Pt}$ from its lattice parameter. The disk specimens were annealed in evacuated quartz capsules at $1000 \mathrm{~K}$ for $10.8 \mathrm{ks}$ to promote disordering, followed by fast quenching into iced brine. They were electropolished to electron transparency in an electrolyte of $\mathrm{HCL}: \mathrm{HNO}_{3}: \mathrm{C}_{2} \mathrm{H}_{5} \mathrm{OH}=4: 1: 5$ by the Ballmann method. In situ observation was carried out with an electron microscope JEM-200CX equipped with a heating stage. The specimen was heated in the microscope once up to $950 \mathrm{~K}$ which is within the temperature region for the Al-phase, and then cooled down to the intended temperature. Since TEM images drifted quickly, they were recorded on imaging plates (IP) and/or video tapes. The dark field images were processed with digitalization of signals in order to improve the quality of image contrast.

\section{Results}

Figure la shows an electron diffraction (ED) pattern taken at $950 \mathrm{~K}$, where one can apparently see diffuse scattering. The scattering is almost the same in intensity 

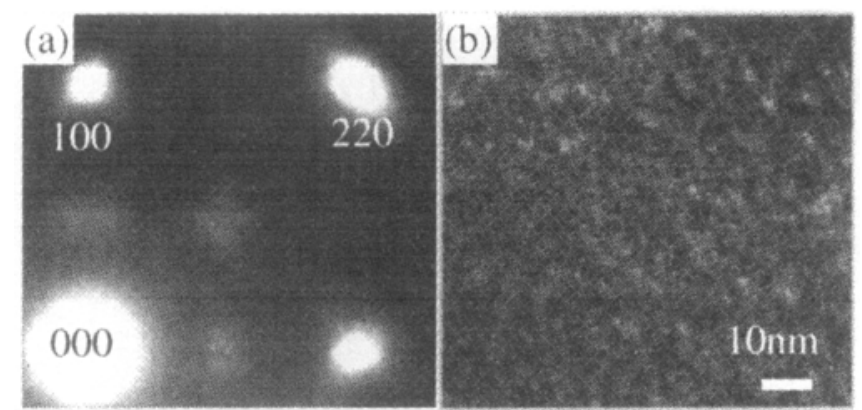

Figure 1. In situ observation of diffuse scattering in electron diffraction pattern and dot-contrast in dark field image $(950 \mathrm{~K})$. distribution as observed for the quenched specimen of this alloy (Ohshima and Watanabe 1973; Kuwano et al 1987). Figure 1b shows a dark field (DF) image taken by using the diffuse scattering around $h k l=110$. In the DF image a fine dot-contrast is observed. These results indicate that the alloy has an SRO state in thermoequilibrium in the temperature region of Al-phase and that the SRO structure is essentially the same as observed in the quenched alloy (Kuwano et al 1987).

Figure 2 shows series of DF images taken at certain intervals at particular temperatures. It is seen in the series (a) $949 \mathrm{~K}$, that a bright dot appears in circle A, but in turn, another dot disappears from circle B within
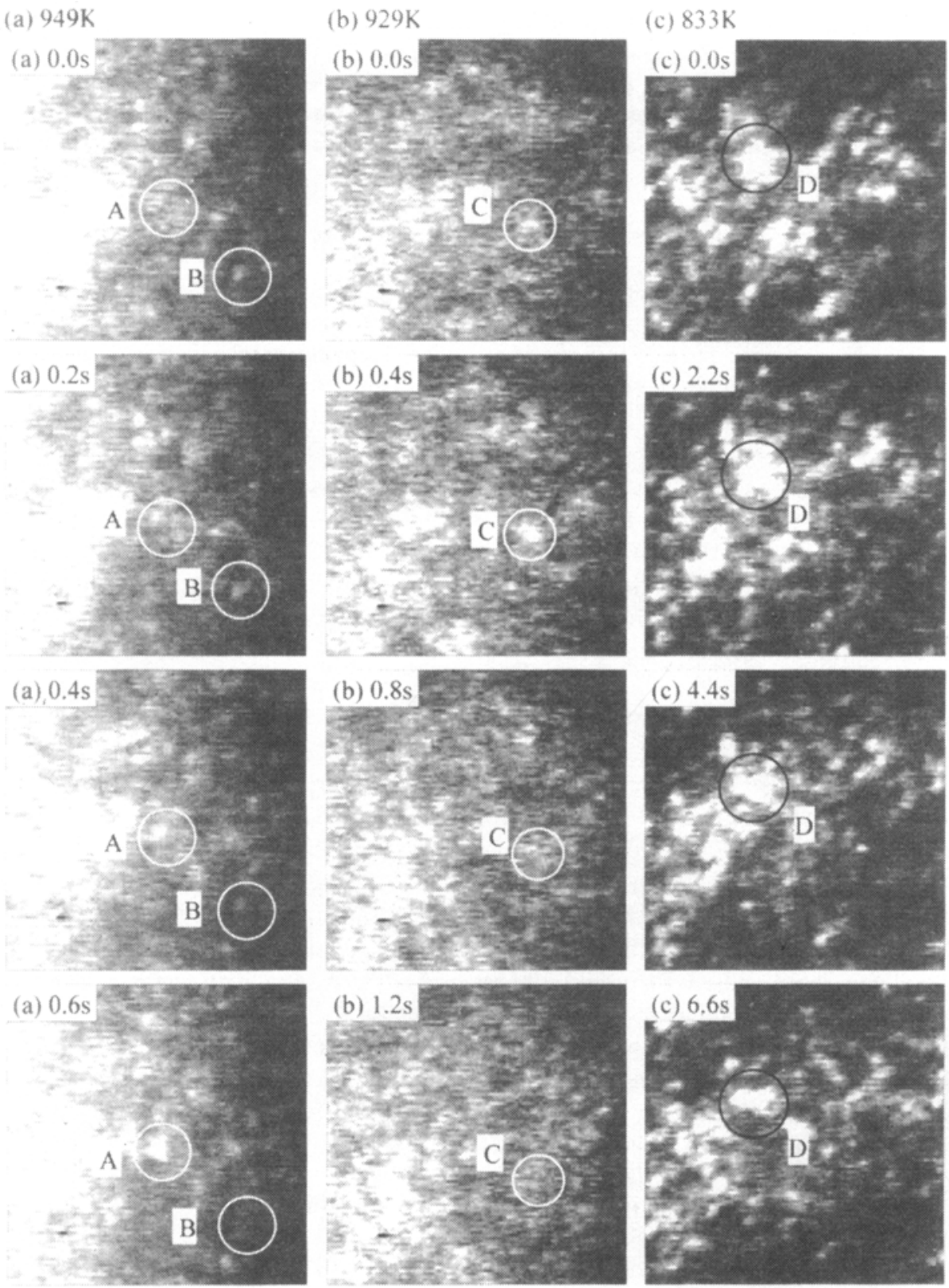

Figure 2. Series of dark field images taken in situ at certain intervals (a) $949 \mathrm{~K}$, (b) $929 \mathrm{~K}$ and (c) $833 \mathrm{~K}$. 
about $0.6 \mathrm{~s}$ : the dot-contrast is flickering very quickly. In series (b) for $929 \mathrm{~K}$, the rate of flickering is lowered so that a dot disappears from circle $\mathrm{C}$ in $1.2 \mathrm{~s}$, and at last a dot remains in circle D after $6.6 \mathrm{~s}$ in (c) $833 \mathrm{~K}$. The bright dots in DF images formed by diffuse scattering do not always correspond to 'microdomains' one by one. However, the rather sharp dot-contrast is thought to indicate an inhomogeneity in the degree of order in the alloy: The degree of order is not constant over the alloy, but has a fluctuation spatially. 'Microdomains' correspond to particular parts where the degree of order happens to be high compared with that in the matrix. The origin of flickering dot-contrast is controversial. Contamination on the specimen surface gives diffuse scattering, and white noise contrast due to the contamination is sometimes flickering in dark field image. If it were the case, the dot-contrast would continue flickering at $833 \mathrm{~K}$ which is still the high temperature and would not grow to an LRO-domain at a lower temperature as shown in figure 3. Bending of the specimen and small variation in observation conditions change the brightness of the dots. But the characteristics of the flickering suggest that the flickering is due to thermal fluctuation inside the specimen (Chiwata et al 1998), i.e. the degree of order has also a temporal fluctuation. The rate of temporal fluctuation decreases with falling temperature, and finally freezes even in the temperature region of $\mathrm{A} 1$.

Figure 3 shows a series of DF images taken to $813 \mathrm{~K}$, indicating the development of a microdomain to a precipitate of the LRO phase of $\mathrm{Ll}_{2-s}$ which has a long

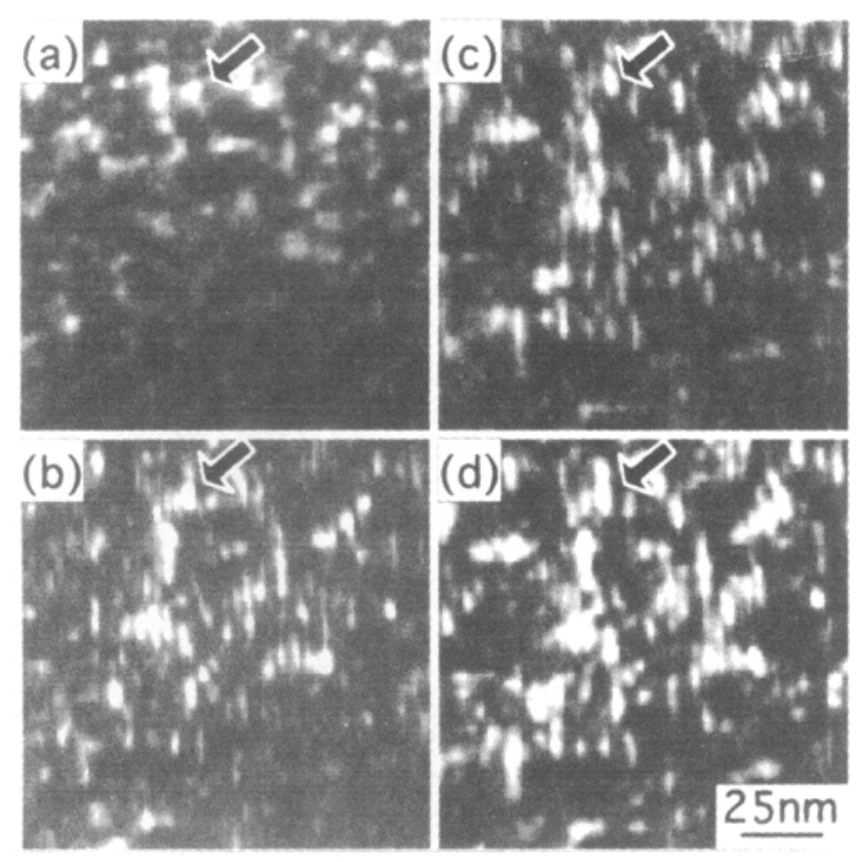

Figure 3. A series of dark field images taken in situ at $813 \mathrm{~K}$ : (a) $4.2 \mathrm{ks}$, (b) $7.8 \mathrm{ks}$, (c) $11.0 \mathrm{ks}$ and (d) $18.7 \mathrm{ks}$. A microdomain (arrowed) grows to a precipitate of $\mathrm{Ll}_{2-s}$ period superstructure derived from $\mathrm{L}_{2}$. The specimen was heated once up to a high temperature to promote disordering in the electron microscope and then held at $813 \mathrm{~K}$. In (a), bright dots are dispersed randomly, but later, some of them (arrowed) grow to larger domains which contain periodic antiphase boundaries.

\section{Discussion}

Figure 4 shows a partial phase diagram for the $\mathrm{Cu}-\mathrm{Pt}$ alloy system based on previous reports (Ogawa et al 1973; Kuwano et al 1987). The $\mathrm{Cu}_{1-x} \mathrm{Pt}_{x}$ alloy with $x \sim 0.25$ has thermoequilibrium phases of $\mathrm{A} 1$ at high temperatures and of the LRO phases ( $\mathrm{Ll}_{2}$, and $\mathrm{L1}_{2-s}$ ) at low temperatures. There is a region of mixed phase of $\left(\mathrm{A} 1+\mathrm{Ll}_{2-s}\right)$. With a consideration of the present experimental results, the region of $\mathrm{A} 1$ is divided into some subregions. At very high temperatures in the Alregion, the alloy has an almost completely disordered state, A1 (CD), where the degree of order is, in this case, defined only statistically and naturally is very small in magnitude. In an intermediate temperature region, A1 (SRO-T), the SRO is developed in a fashion of 'microdomains'. The degree of order is not constant, but fluctuates spatially and temporally in the alloy. At a lower temperature in Al (SRO-S), however, the degree of order fluctuates just spatially. The phase boundaries between Al (CD) and Al (SRO-T) and between Al (SRO-T) and A1 (SRO-S) have a certain width since the state is altered gradually and continuously to the other. These subregions do not always exist in $\mathrm{A} 1$ region of another alloy system. The alloy of $\alpha-\mathrm{Cu}_{1-x} \mathrm{~A} 1_{x}$ with $x \sim 0.19$ has the phase relation similar to that of the present $\mathrm{Cu}-\mathrm{Pt}$ alloy; it has the states of $\mathrm{A} 1$ and $\mathrm{A} 1+\mathrm{LRO}$,

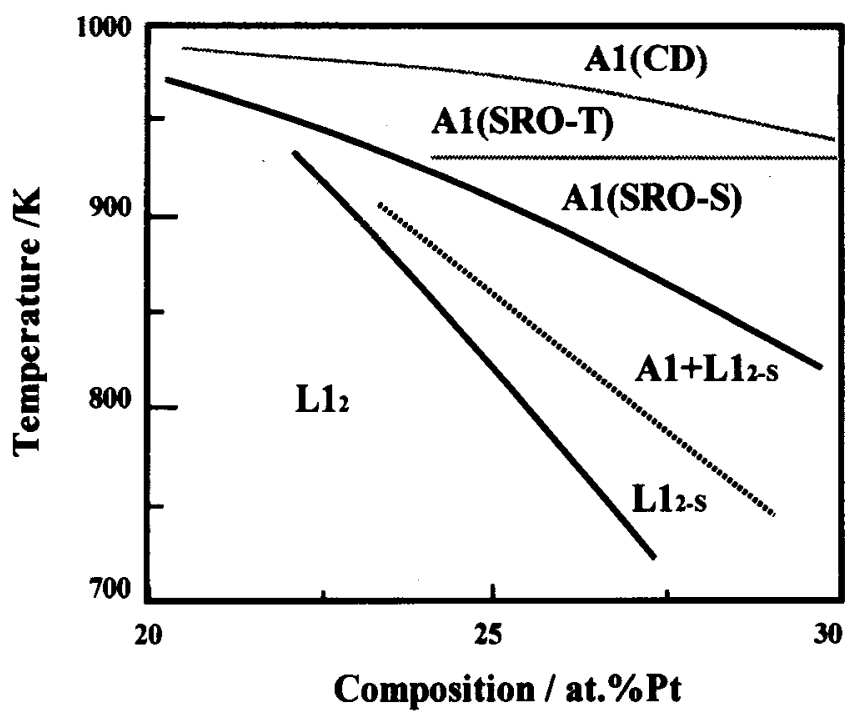

Figure 4. Partial phase diagram for $\mathrm{Cu}-\mathrm{Pt}$. The $\mathrm{Al}$ region is divided into subregions. 
and the A1-phase also has SRO. The LRO phase has a long period antiphase structure derived from $\mathrm{L1}_{2}$ (Gaudig and Warlimont 1969; Kuwano et al 1977). However, the ordering temperature is lower than $\sim 635 \mathrm{~K}$ and then at high temperature the degree of order decreases vanishingly before it starts to have temporal fluctuation; the A1-phase of $\mathrm{Cu}-\mathrm{A} 1$ does not have a subregion of $\mathrm{Al}$ (SRO-T). The results for $\mathrm{Cu}-\mathrm{Al}$ alloy will be described separately.

Thermodynamically, the transformation of $\mathrm{Ll}_{2-s} \Leftrightarrow \mathrm{Al}$ is of a first order, and therefore the formation of $\mathrm{L}_{2-s}$ in $\mathrm{A} 1$ was thought to proceed by the nucleation and growth process. But actually, there are already microdomains in the matrix of A1-phase as the thermoequilibrium state, and some of the microdomains play a role of nucleates of the LRO phase. The formation of LRO phase therefore proceeds almost continuously via the SRO state without a process of nucleation. Conventionally, the phase boundary between (LRO + A1) and $A 1$ is defined as a border-line for the LRO phase to exist stably with assumption of coexistence of large domains of LRO and the matrix of A1. It should be noted that in the present case of transformation from Al to $(\mathrm{A} 1+\mathrm{LRO})$, the boundary of $(\mathrm{A} 1+\mathrm{LRO}) / \mathrm{A} 1$ is regarded as the border for the microdomains in $A 1$ (SRO) to start growing infinitely. In this case, additional effects such as elastic energy between microdomains and the matrix are expected to have significant influences on the stability of phases. Then the location of the boundary of (A1 + LRO)/A1 in the phase diagram depends on the manner of transformation and on the microstructure in the alloy.

\section{Conclusions}

From the in situ observation of the SRO state and of the ordering process to $\mathrm{LRO}$ phase in $\mathrm{Cu}_{3} \mathrm{Pt}$, the following results were obtained.

(I) The SRO state in $\mathrm{Cu}_{3} \mathrm{Pt}$ can be interpreted with a microdomain model. The SRO is stable at a high temperature.

(II) At high temperatures, the degree of order fluctuates temporally as well as spatially. The temporal fluctuation in degree of order stops at a lower temperature.

(III) Since some of microdomains can become seeds of the LRO phase, the transformation to LRO proceeds almost continuously without a process of nucleation in spite of which the transformation is of a first order.

(IV) The region of $\mathrm{A} 1$ in the phase diagram is divided into three subregions depending on the existence of temporal fluctuation and spatial one in the degree of SRO.

\section{Acknowledgements}

This work was partly supported by Grant-in-Aid for Scientific Research on the Priority Area 'Investigation of Microscopic Mechanism of Phase Transformations for the Structure Control of Materials' (No. 09242106), and by Grant-in-Aid for Scientific Research (B) (No. 10450243) from the Ministry of Education, Science, Sports and Culture, Japan.

\section{References}

Aubauer H P 1972 Acta Metall. 20 165, 173

Chevalier J-P and Stobbs W M 1979 Acta Metall. 27285

Chiwata N, Sakai T, Matsumoto A, Kuwano N and Oki K 1998 Electron microscopy, in Proc. 14 int. cong. EM, Cancun (eds) H A Calderón Benavides and M J Yacamán (Mexico: Inst. of Phys. Pub.) vol. III, p. 27

Gaudig W and Warlimont H 1969 Z. Metallk. 60488

Krivoglaz M A 1969 Theory of X-ray and thermal neutron scattering by real crystals (New York: Plenum Press) p. 169

Kuwano N, Doi T and Eguchi T 1977 Trans. JIM 18488

Kuwano N, Nakayama R and Oki K 1987 Trans. JIM 281

Lee K-H, Hiraga K and Hirabayashi M 1990 Mater. Trans. JIM 31449

Moss S C 1969 Phys. Rev. Lett. 221108

Ogawa S, Iwasaki H and Terada A 1973 J. Phys. Soc. Jap. 34 384

Ohshima K and Watanabe D 1973 Acta Crystallogr. A29 520

Torfs E, Stals L, Van Landuyt J, Delavignette P and Amelinckx S 1974 Phys. Status Solidi 2245

Torfs E, Van Landuyt J, Stals L and Amelinckx S 1975 Phys. Status Solidi 31633 